\title{
Obesity Paradox in Amputation Risk Among Nonelderly Diabetic Men
}

\author{
Min-Woong Sohn ${ }^{1,2}$, Elly Budiman-Mak ${ }^{1,3}$, Elissa H. Oh², Michael S. Park ${ }^{4}$, Rodney M. \\ Stuck $^{5,6}$, Neil J. Stone ${ }^{7}$, and William B. Pearce ${ }^{4}$ \\ ${ }^{1}$ Center for Management of Complex Chronic Care, Hines Veterans Affairs Hospital, Hines, \\ Illinois, USA \\ ${ }^{2}$ Institute for Healthcare Studies, Feinberg School of Medicine, Northwestern University, Chicago, \\ Illinois, USA \\ ${ }^{3}$ Department of Medicine, Loyola University Stritch School of Medicine, Maywood, Illinois, USA \\ ${ }^{4}$ Department of Surgery, Feinberg School of Medicine, Northwestern University, Chicago, Illinois, \\ USA \\ ${ }^{5}$ Surgical Service, Hines Veterans Affairs Hospital, Hines, Illinois, USA \\ ${ }^{6}$ Department of Orthopedic Surgery, Loyola University Stritch School of Medicine, Maywood, \\ Illinois, USA \\ ${ }^{7}$ Department of Medicine, Feinberg School of Medicine, Northwestern University, Chicago, \\ Illinois, USA
}

\section{Abstract}

The association between BMI and amputation risk is not currently well known. We used data for a cohort of diabetic patients treated in the US Department of Veterans Affairs Healthcare System in 2003. Men aged $<65$ years at the end of follow-up were examined for their amputation risk and amputation-free survival during the next 5 years (2004-2008). Compared to overweight individuals (BMI $25-29.9 \mathrm{~kg} / \mathrm{m}^{2}$ ), the risks of amputation and treatment failure (amputation or death) were higher for patients with BMI $<25 \mathrm{~kg} / \mathrm{m}^{2}$ and were lower for those with BMI $30 \mathrm{~kg}$ / $\mathrm{m}^{2}$. Individuals with BMI $\geq 40 \mathrm{~kg} / \mathrm{m}^{2}$ were only half as likely to experience any (hazard ratios $(\mathrm{HR})=0.49 ; 95 \%$ confidence interval $(\mathrm{CI}), 0.30-0.80)$ and major amputations $(\mathrm{HR}=0.53 ; 95 \%$ CI, 0.39-0.73) during follow-up as overweight individuals. While the amputation risk continued to decrease for higher BMI, amputation-free survival showed a slight upturn at BMI $>40 \mathrm{~kg} / \mathrm{m}^{2}$. The association between obesity and amputation risk in our data shows a pattern consistent with "obesity paradox" observed in many health conditions. More research is needed to better understand pathophysiological mechanisms that may explain the paradoxical association between obesity and lower-extremity amputation (LEA) risk.

Lower-extremity amputation (LEA) is an adverse outcome with significant morbidity and mortality sequellae, and amputation-free survival is the main goal of treatment for patients

\footnotetext{
(C) 2011 The Obesity Society

Correspondence: Min-Woong Sohn (Min-Woong.Sohn@va.gov).

DISCLOSURE

The authors declared no conflict of interest.

The corresponding author had full access to all of the data in the study and takes responsibility for the integrity of the data and the accuracy of the data analysis.
} 
with peripheral arterial disease (PAD) and/or foot ulcers. In a previous study, we reported a significant $\mathrm{J}$-shaped association between BMI and foot ulceration risk (1), which suggests that the association between BMI and LEA risk might also show a similar pattern with the risk of LEA increasing as the BMI increases among obese individuals. The objective of this study is to examine the relationship between BMI and 5-year LEA risk for nonelderly men with diabetes.

\section{METHODS AND PROCEDURES}

\section{Study cohort}

We used inpatient and outpatient datasets (2) for fiscal year 2003 (October 2002-September 2003) to identify the study cohort. These datasets contain all inpatient and outpatient administrative records for the US Department of Veterans Affairs hospitals and clinics. The study population consists of all male individuals who had a diagnosis of diabetes in 2003 and were $<65$ years of age at the end of follow-up. We excluded from the study patients who died before 1 October 2003, who had a history of lower-extremity amputations in 19972003, who were new users or new enrollees in 2003, and who did not have at least one measure of both height and weight in 2003.

\section{Variables}

Two study outcomes are an LEA and amputation-free survival identified by any occurrence of LEA or death. We found all minor and major LEAs in inpatient and outpatient medical records between 2004 and 2008 using ICD-9-CM and CPT-4 codes published elsewhere (3). Death dates were obtained from the Veterans Affairs Vital Status file (4).

We obtained all height and weight measures taken in 2003 for our study cohort and computed the average BMI, $\mathrm{kg} / \mathrm{m}^{2}$. Patients were placed into groups that are divided by 5unit BMI increments as defined under World Health Organization BMI classification: normal (20-24.9 kg/m²), overweight ( $\left.25-29.9 \mathrm{~kg} / \mathrm{m}^{2}\right)$, class I obesity $\left(30-34.9 \mathrm{~kg} / \mathrm{m}^{2}\right)$, class II obesity $\left(35-39.9 \mathrm{~kg} / \mathrm{m}^{2}\right)$, and class III obesity ( $40 \mathrm{~kg} / \mathrm{m}^{2}$ or higher).

Other individual, facility, and geographic factors that are likely to confound the association between weight and study outcomes were identified. Diabetes duration was estimated as the number of years a patient was treated for diabetes in the Veterans Affairs. Diabetes control was measured by the average of hemoglobin diabetes control measures taken in 2003 . We also included diabetic complications such as foot ulcers, peripheral arterial disease, peripheral neuropathy, and Charcot arthropathy, found in 2003 administrative records in the analysis.

The albumin and low-density lipoprotein levels measured in the baseline year were also obtained as measures of vascular and nutritional health.

\section{Statistical analysis}

For each patient, we computed the time to an LEA and the time to an LEA or death from the index date (1 October 2003) that occurred during the 5-year follow-up. For persons with multiple LEAs, we use the first LEA as our endpoint. The time to event variables were analyzed using Cox proportional hazards regressions to estimate the risk of amputation or amputation-free survival associated with each BMI group, adjusting for baseline diabetes duration, severity, and control, patient demographic characteristics and comorbidities, and measures of vascular and nutritional health in the baseline year. 


\section{RESULTS}

There were 115,226 men in our study cohort, of whom 597 (0.52\%) had experienced minor or major amputations, $280(0.24 \%)$ experienced major amputations, and 13,016 (11.3\%) experienced a combined endpoint of any amputation or death (treatment failure) during a mean follow-up of 4.5, 4.7, and 4.7 years, respectively. These represent 0.11 amputations, 0.05 major amputations, and 2.47 treatment failures per 100 person-years. Over two thirds of the cohort (64\%) were obese (BMI $230 \mathrm{~kg} / \mathrm{m}^{2}$ ) and almost $13 \%$ class III obese (BMI $\geq 40$ $\left.\mathrm{kg} / \mathrm{m}^{2}\right)$.

Figure 1 shows relative risks of any LEA, major LEA, and any treatment failure, adjusted for potential confounders. Compared to those who were overweight (BMI 25-29.9 kg/m 2 ), patients with BMI $<20$ and $20-24.9 \mathrm{~kg} / \mathrm{m}^{2}$ were $92 \%$ (hazard ratios $(\mathrm{HR})=1.92 ; 95 \%$ confidence interval (CI), 1.16-3.20; $P=0.01$ ) and 33\% (HR $=1.33 ; 95 \% \mathrm{CI}, 1.03-1.72 ; P=$ $0.03)$ more likely to have any LEA and 3.1 times $(\mathrm{HR}=3.11 ; 95 \% \mathrm{CI}, 1.67-5.78 ; P<0.01)$ and 1.7 times $(\mathrm{HR}=1.67 ; 95 \% \mathrm{CI}, 1.17-2.36 ; P=0.01)$ more likely to have a major LEA, respectively.

The class I obese (BMI $30-34.9 \mathrm{~kg} / \mathrm{m}^{2}$ ) were not significantly different from overweight patients in the risk of having any or major amputations. The class II obese were about $40 \%$ lower in risks of both any $(\mathrm{HR}=0.61 ; 95 \% \mathrm{CI}, 0.46-0.81 ; P<0.01)$ and major $(\mathrm{HR}=0.58$; 95\% CI, $0.38-0.88 ; P=0.01)$ LEAs. The class III obese were almost half as likely to have any $(\mathrm{HR}=0.53 ; 95 \% \mathrm{CI}, 0.39-0.73 ; P<0.01)$ or major $(\mathrm{HR}=0.49 ; 95 \% \mathrm{CI}, 0.30-0.80 ; P<$ $0.01)$ LEAs, compared to the overweight patients.

Amputation-free survival increased as BMI increased. Compared to overweight individuals, individuals with BMI $<20 \mathrm{~kg} / \mathrm{m}^{2}$ were three times more likely (HR $=3.03 ; 95 \%$ CI, 2.73$3.76 ; P<0.01)$ and individuals with BMI $20-24.9 \mathrm{~kg} / \mathrm{m}^{2}$ were $53 \%$ more likely to experience any treatment failure ( $\mathrm{HR}=1.53 ; 95 \% \mathrm{CI}, 1.44-1.624 ; P<0.01)$ during followup. The lowest risk of treatment failure was found for individuals with class I and II obesity and the upturn at the class III obesity was statistically significant $(\mathrm{HR}=1.22$; $95 \% \mathrm{CI}, 1.14$ 1.306; $P<0.01$ ) compared to individuals with class II obesity.

\section{DISCUSSION}

Our results show that higher BMI was associated with lower 5-year risk of any LEA and major LEA for diabetic men aged $<65$ years. The LEA risk decreased monotonically as the BMI increased throughout the entire BMI spectrum.

Our results are surprising and "paradoxical," because there is an extensive body of literature that obesity is associated with adverse health outcomes in the general population $(5,6)$ as well as among diabetic patients (7). Furthermore, we recently showed a significant J-shaped association between BMI and foot ulceration with class III obese being twice more likely to develop foot ulceration during a 5-year follow-up than overweight individuals (1). Because foot ulcers are by far the most important risk factor for LEA, our results that showed monotonically decreasing LEA risk with increasing BMI are unexpected.

This is the first study that shows an obesity paradox in LEA risk. We do not have a good explanation for this paradox or know whether apparent protective effects of class III obesity have any pathophysiological basis. A recent study by Biasucci et al. showed that severe obesity might be protective of vascular function through increased levels of endothelial progenitor cells (8). The Biasucci et al. study was based on nondiabetic individuals and we do not know whether this finding would equally apply to diabetic patients who are known to have reduced numbers of endothelial progenitor cells (9-11). If it proves to be the case, one 
can further hypothesize that, even though severely obese individuals experience more foot ulceration, they have better wound healing than individuals with lower body mass through better mobilization of endothelial progenitor cells that are activated with skin breach $(12,13)$. This can potentially explain the apparent paradox between the increased foot ulceration risk and decreased amputation risk among severely obese individuals. Future studies need to examine whether severe obesity is associated with better wound healing among diabetic individuals.

The elevated risk for individuals with underweight (BMI <20) is hardly surprising. Individuals in this group have higher risk of foot ulceration than those with normal weight (1) and may be more likely to experience (or have experienced) "wasting" as a result of longstanding diabetes, insulin use, or tobacco exposure. In our study, we were able to control for both diabetes duration and insulin use at baseline but smoking data were not available.

A limitation of the study includes the use of administrative data whose accuracy of disease and procedure coding is not clearly known. Because there is no validation study on the accuracy of our amputation data, we compared them to national estimates from the Center for Disease Control and Prevention (http://www.cdc.gov/diabetes/statistics/lealevel/ table8_age.htm, Accessed 20 April 2011). While the 1-year unadjusted amputation rate for our cohort was 5.2/1,000, the national rate for diabetic population $<65$ years old were $5.1 / 1,000$, which suggests that our data reasonably resembled the national data on amputations.

In conclusion, our data show that higher BMI is associated with lower 5-year LEA risk among nonelderly diabetic men. After adjusting for baseline glucose level, nutritional status, diabetes severity, and comorbidities, we found that individuals with BMI $\geq 40 \mathrm{~kg} / \mathrm{m}^{2}$ are almost half as likely to have any or major LEA in the next 5 years than those with BMI 25$29.9 \mathrm{~kg} / \mathrm{m}^{2}$. We do not currently have good explanations for this paradoxical association but suggested some possible mechanisms.

\section{Acknowledgments}

The authors gratefully acknowledge the financial support from the Center for Management of Complex Chronic Care, Hines VA Hospital, Hines, IL (LIP 42-099; E.B-M., MD, Principal Investigator). The paper presents the findings and conclusions of the authors; it does not necessarily represent the Department of Veterans Affairs or Health Services Research and Development Service.

\section{References}

1. Sohn MW, Budiman-Mak E, Lee TA, Oh E, Stuck RM. Significant J-shaped association between body mass index (BMI) and diabetic foot ulcers. Diabetes Metab Res Rev. 2011; 27:402-409. [PubMed: 21360633]

2. Murphy PA, Cowper DC, Seppala G, Stroupe KT, Hynes DM. Veterans Health Administration inpatient and outpatient care data: an overview. Eff Clin Pract. 2002; 5:E4. [PubMed: 12166925]

3. Sohn MW, Stuck RM, Pinzur M, Lee TA, Budiman-Mak E. Lower-extremity amputation risk after charcot arthropathy and diabetic foot ulcer. Diabetes Care. 2010; 33:98-100. [PubMed: 19825822]

4. Sohn MW, Arnold N, Maynard C, Hynes DM. Accuracy and completeness of mortality data in the Department of Veterans Affairs. Popul Health Metr. 2006; 4:2. [PubMed: 16606453]

5. Flegal KM. Excess deaths associated with obesity: cause and effect. Int J Obes (Lond). 2006; 30:1171-1172. [PubMed: 16871273]

6. Flegal KM, Graubard BI, Williamson DF, Gail MH. Excess deaths associated with underweight, overweight, and obesity. JAMA. 2005; 293:1861-1867. [PubMed: 15840860] 
7. Batty GD, Kivimaki M, Smith GD, Marmot MG, Shipley MJ. Obesity and overweight in relation to mortality in men with and without type 2 diabetes/impaired glucose tolerance: the original Whitehall Study. Diabetes Care. 2007; 30:2388-2391. [PubMed: 17623818]

8. Biasucci LM, Graziani F, Rizzello V, et al. Paradoxical preservation of vascular function in severe obesity. Am J Med. 2010; 123:727-734. [PubMed: 20670727]

9. Loomans CJ, de Koning EJ, Staal FJ, et al. Endothelial progenitor cell dysfunction: a novel concept in the pathogenesis of vascular complications of type 1 diabetes. Diabetes. 2004; 53:195-199. [PubMed: 14693715]

10. Fadini GP, Agostini C, Avogaro A. Endothelial progenitor cells and vascular biology in diabetes mellitus: current knowledge and future perspectives. Curr Diabetes Rev. 2005; 1:41-58. [PubMed: 18220581]

11. Fadini GP, Miorin M, Facco M, et al. Circulating endothelial progenitor cells are reduced in peripheral vascular complications of type 2 diabetes mellitus. J Am Coll Cardiol. 2005; 45:1449_ 1457. [PubMed: 15862417]

12. Brem H, Tomic-Canic M. Cellular and molecular basis of wound healing in diabetes. J Clin Invest. 2007; 117:1219-1222. [PubMed: 17476353]

13. Liu ZJ, Velazquez OC. Hyperoxia, endothelial progenitor cell mobilization, and diabetic wound healing. Antioxid Redox Signal. 2008; 10:1869-1882. [PubMed: 18627349] 


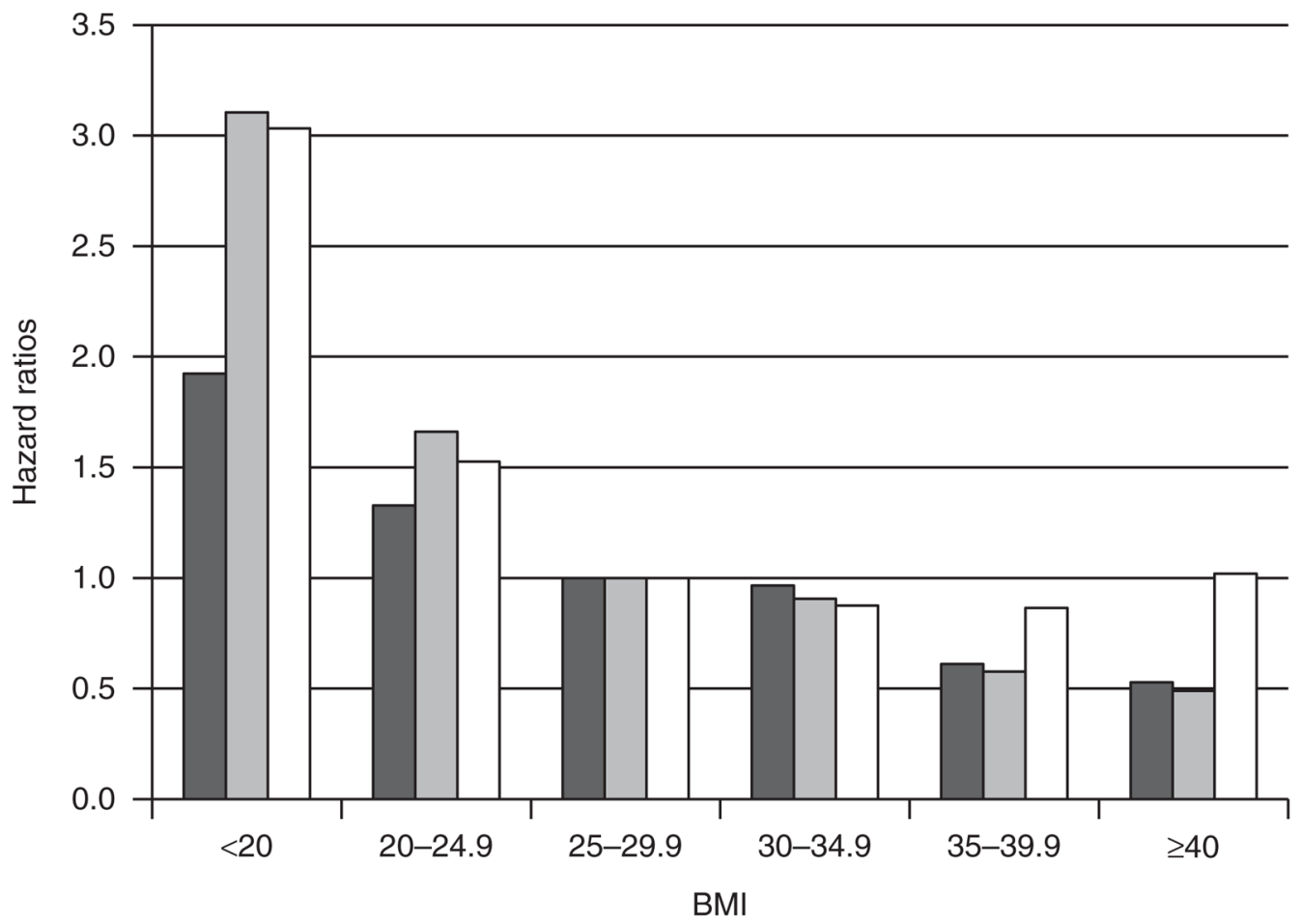

BM

Any amputation $\quad \square$ Major amputation $\quad \square$ Amputation or death

Figure 1.

Relative risks of adverse outcomes by baseline BMI. Adjusted for age, race/ethnicity, marital status, diabetes medication use, diabetes duration, diabetes control (A1c), albumin, comorbidities (peripheral arterial disease, peripheral neuropathy, foot ulcers, congestive heart failure, valve disorders, paralysis, renal failure, electrolytes and fluid disorders, and deficiency anemias), and indicators of Veteran Integrated Service Network (VISN). 